\title{
Ethical dilemmas encountered in suicide research and management: Experiences of young mental health professionals
}

\author{
SUPREET KAUR BHASIN, ISHITA U BHARADWAJ, R P BENIWAL, VANYA GUPTA, TRIPTISH BHATIA, SMITA N DESHPANDE
}

\begin{abstract}
This study aimed to explore ethical dilemmas and challenges faced by young mental healthcare researchers and professionals working with survivors of suicide (hereinafter suicide survivors). Two focus group discussions (FGDs) Researcher FGD (with those engaged in suicide research) and Clinician FGD (with those providing treatment to suicide survivors) - consisting of openended questions and lasting for 70-90 minutes were conducted and analysed using Thematic Analysis. Five themes were identified: i) struggling with the incongruity of harm within benefit, ii) difficulty in delineating boundaries, iii) self-doubt in one's professional competence, iv) nature of suicide straining the limits of confidentiality, and v) working with structural limitations. The study helps to understand the obstacles and dilemmas encountered in adhering to ethical principles while working with vulnerable individuals.
\end{abstract}

Keywords: Ethical dilemmas, suicide, thematic analysis, mental healthcare professionals, focus group discussions

\section{Introduction}

Attempts to die by suicide are a grave public concern due to their complex nature and increasing magnitude. Globally, $8,00,000$ individuals die every year by suicide (1), with the highest number of suicide deaths in Southeast Asia being recorded in India (thus labelled the "suicide capital of Southeast Asia") (2). The global suicide crisis has not been adequately addressed through research and policy

Authors: Supreet Kaur Bhasin (supreetbhasin293@gmail.com), Department of Psychology, University of Delhi, INDIA; Ishita U Bharadwaj (ishipsycho@gmail.com), Department of Psychology, University of Delhi, INDIA; RP Beniwal (beniwal_9@yahoo.co.in) Centre of Excellence in Mental Health, ABVIMS-Dr. Ram Manohar Lohia Hospital, New Delhi, 110001,INDIA; Vanya Gupta (vanyaguptagzb@gmail.com) Healing Self, Ghaziabad, Uttar Pradesh, INDIA; Triptish Bhatia (bhatiatriptish@yahoo.co.in), Indo-US Projects, Centre of Excellence in Mental Health, ABVIMS-Dr. Ram Manohar Lohia Hospital, New Delhi, 110001, INDIA; Smita N Deshpande (corresponding author smitadeshp@gmail.com), Centre of Excellence in Mental Health, ABVIMSDr. Ram Manohar Lohia Hospital, New Delhi, 110001, INDIA.

To cite: Bhasin SK, Bharadwaj IU, Beniwal RP, Gupta V, Bhatia T, Deshpande SN. Ethical dilemmas encountered in suicide research and management: Experiences of young mental health professionals. Indian J Med Ethics. Published online on December 15, 2021.DOI: 10.20529/IJME.2021.096

Manuscript Editor: Mala Ramanathan

Abida Sultana and an anonymous reviewer.

(c) Indian Journal of Medical Ethics 2021 interventions (3), probably because research and clinical management raise several ethical challenges $(4,5)$.

Conducting suicide research poses complex challenges both for researchers and participants (6) because suicide research tends to focus on safeguarding participants and ignore the vulnerability of researchers $(7,8)$. Vulnerability comprises emotional responses, depending on being in a particular position (9). In research centred on sensitive issues, there is a need for protecting the researchers too, considering their sociocultural background, academic training, specific features of the research and its setting (10). When dealing with sensitive issues such as suicide, the participants are intrinsically vulnerable, either due to mental health symptoms (pain, hopelessness etc) or due to inadequate resources and support. This multiplies the ethical challenges and burden on the researchers (11) adding to their vulnerability. The ethical dilemmas range from selecting an appropriate study design to developing effective safeguard mechanisms for everyone involved (11). Some ethical dilemmas include potential harm to both the researcher and participant, blurred boundaries while acting as a facilitator in the research process and maintaining confidentiality (12-14). The most common potential harms to researchers are survivor guilt, distress and burden due to heightened sense of responsibility towards those who attempted or died by suicide (12).

Clinician ethical dilemmas regarding patients with suicidal thoughts are less discussed - most publications being practitioner opinion surveys $(15,16)$. Prominent clinician dilemmas are confidentiality concerns, limited direct care of patients with suicidal thoughts, and clouding of personal and professional domains (17-19). Thus, ethical dilemmas and challenges encountered by mental healthcare professionals, especially younger professionals involved in suicide research and clinical management, need closer examination.

The present study aimed to explore the nature of ethical dilemmas encountered by researchers and also by mental health professionals engaging with suicide survivors.

\section{Methods}

This study describes an amalgamation of the results of three sub-investigations - two Focus Group Discussions (FGDs) involving suicide researchers and clinicians involved in managing suicide survivors, and a reflexive analysis of the first author's qualitative research experience as part of her $\mathrm{PhD}$ 
dissertation with suicide survivors. The study was held at a tertiary care government teaching institution in the academic year 2019-20. The study was embedded in a larger study titled "Multi-centric randomised controlled trial to evaluate the efficacy of telephone based psychosocial interventions on future suicide risk in suicide attempters" Both this study and the larger study were reviewed and approved by the Institutional Ethics Committee of the institution vide letter numbers 316(34/2019)/IEC/ABVIMS/ RMLH) 5516/19 and / 189 (08/2017) /IEC /ABVIMS / RMLH/ 901 , respectively.

\section{FGD Members}

Thirteen young mental health workers with postgraduate qualifications working at this centre were recruited for the FGDs through purposive and snowball sampling. Purposive sampling is a form of non-probability sampling in which researchers rely on their own judgement when choosing members of the population to participate in their surveys. Snowballing - a type of purposive sampling - involves one participant recommending several other people who meet the inclusion criteria, so the participant list can grow (20).

Our participants were from diverse backgrounds in terms of work engagement, sex, and experience with suicide survivors, as well as their social status and culture. Each was personally contacted, informed, and invited to participate in person and on the phone. Consent was obtained.

\section{Researcher FGD (FGD 1)}

This included five young mental health professionals (2 clinical psychologists and 3 psychiatric social workers (henceforth referred to as RF), currently working as Research Fellows in an Indian Council of Medical Research (ICMR) funded research project and possessing research experience with suicide survivors. To our knowledge, this is one of the few papers examining ethical research undertaken by these research fellows which was a randomised controlled trial (RCT) to evaluate the efficacy of telephone-based psychosocial interventions on future suicide risk in suicide survivors $(21,22)$.

As part of their protocol, they had to adhere to the study plan of work and not provide any extra intervention. Their comparator group was involved in telephone interventions in which they had only to ask about treatment compliance, offer general information, and not provide any coping strategies. Providing clinical care was not part of their duties. However, prior to inclusion in the RCT, it was ensured by the RFs that their participant-survivors had fully recovered physically from their injuries. The protocol also instructed the research fellows in participant protection. The participant (suicide attempt survivor) could withdraw from the study at any point if they wished. In case he/she felt overwhelmed by suicidal thoughts or was perceived by the RFs to be at risk for suicide during intervention or interview, they were immediately referred to their treating clinician.

\section{Clinician FGD (FGD 2):}

In this, eight mental health professionals, working as Senior Residents (SR, post MD Psychiatry) $(n=4))$ and young Clinical Psychologists (CP, post-MPhil Psychology), $(n=4))$ referred to as Clinicians henceforth, participated. All had provided clinical care to suicide survivors but did not necessarily possess research experience in studying suicide.

Members of both FGDs were psychiatry residents and trained clinical psychologists. They were in regular touch with the senior members of the treatment teams and could discuss any issues with their team members and seniors.

\section{Procedure}

FGD 1, lasting for 90 minutes, focused on dilemmas like role conflict, confidentiality concerns, and the fear of causing harm (breach of non-maleficence) encountered while conducting research with suicide attempt survivors.

FGD 2, lasting for 70 minutes focused on the ethical dilemmas faced in the clinical management of patients who had attempted suicide. Findings from both the FGDs were compared to discover the points of congruence and divergence in the dilemmas encountered by either group.

For both the FGDs, open-ended questions were formulated as anchor points for discussion, borrowing from the first author's reflexive account. The first author was engaged in her research consisting of in-depth interviews with suicide survivors. Various dilemmas, such as an enhanced sense of responsibility combined with fear, difficulty in establishing boundaries, role conflict (clinician or researcher) and others were received in the course of these interviews. The reflexive nature of qualitative research aided in capturing the emerging concerns and emotional discord experienced by the researcher. This motivated the researchers to discuss whether similar concerns had been faced and resolved by other researchers and clinicians engaged in suicide research and management. These reflective points were then shared with experts, including senior psychiatrists and senior research psychologists, and revised to be made more comprehensive. A few examples of the questions are:

- Have you ever found yourself struggling with any form of ethical challenge/dilemma while working with individuals presenting a risk of suicide? Would you like to share some situations where you have encountered ethical questions and concerns during your professional role and practice with such individuals?

- $\quad$ Could you describe some instances where ethical issues have been quite difficult to deal with?

- What role (if any) do you feel our psychosocial and cultural context plays in some of the ethical challenges around suicide? Have you ever encountered any psychosocial dynamics in a case of suicide, which has contributed to simplifying/complicating the ethical 
challenges being dealt with by you?

- Which environmental and institutional factors (if any), do you feel have contributed to the creation of ethical dilemmas?

- Would you like to share any situation, where despite following ethical guidelines, you struggled to resolve an ethical dilemma?

\section{Data recording and analysis}

During both the FGDs, the first author acted as the moderator and participants were encouraged to speak freely and probed when required. The discussions were audio-recorded and notes were made simultaneously by a designated junior research fellow.

The recorded data was transcribed and subjected to analyst triangulation. The authors SKB and VG who trained at the same institution but are currently working in different institutions, sharing a similar ontological and epistemological stance in qualitative research methodology, subjected the data to two levels of coding independently. The first coding cycle employed open coding (for which the transcripts were broken down into discrete parts, and examined for similarities and differences, based on the first impression of the authors); followed by pattern coding (pulling together smaller sets of data to build meaningful explanations and inter-relationships between the discrete parts) in the second cycle (23).

These codes were, subsequently, compared to observe points of convergence, confirmations, and contradictions within the data. The nature of analysis was inductive, and principles of thematic analysis as recommended by Braun and Clarke were followed (24).

Accounts from the transcripts were identified as examples for the developed codes. These were further subjected to critique, re-examination, counterarguments, and repeated questioning from both the analysts. Subsequently, these categories and patterns were reviewed and systematically organised into subthemes. These subthemes were further clustered together to form consequent themes, with their respective operational definitions.

\section{Results}

In the current study, the FGD members were aged 27-33 years, of whom nine were female and four male, with 1-3 years of experience in dealing with suicide survivors. Having acquired their professional qualifications from different parts of the country, they had been residing in Delhi and Delhi-NCR for at least the past two years.

The data was subjected to thematic analysis resulting in seventeen subthemes, subsumed under five themes: (i) struggling with the incongruity of harm within benefit, (ii) difficulty in delineating boundaries, (iii) self-doubt in one's professional competence, (iv) nature of suicide straining the limits of confidentiality, and lastly, (v) working with structural limitations (Table 1).

\section{Theme 1: Struggling with the incongruity of harm within benefit}

While attempting to meticulously document the first person narratives of suicide survivors, and thereby to enrich the study, the researchers have to be mindful of avoiding any harm to the research participants involved. During FGD 1, all the researchers revealed their apprehensions of unintentionally harming their participants while involving them in a sensitive yet standard research process. They expressed their concern that the inflexible and pre-set nature of a standardised research protocol might reinforce symptomatology in an already vulnerable group of people. For instance, some of the RFs shared that:

The assessments are required to ascertain the severity and intensity of suicidal thoughts or hopelessness.... But at times, it gets disturbing for the participants (suicide attempter survivors) to go back down that road, makes them feel edgy and heavy-hearted. So as a researcher, asking these questions make me fearful and apprehensive as well. (RF2)

In addition, the inability to effectively intervene with tailormade research interventions also generated doubts about the benefit to the participants (suicide survivors).

Suicidal ideation can have different purposes ... for a person with dissociation or personality disorder or depression, the reasons for suicide are different, but the treatment is the same .... So, in some disorders, we are reinforcing the problem instead of helping them. (RF3)

Another reason cited for this ethical dilemma was that, while the researchers were expected to establish a deep personal connect with the participants/suicide survivors, it was mandatory for them to refrain from giving in to their participant's expectations of the researcher becoming their counsellor. One of the researchers, shedding light on how refraining from such dual relationships in research was necessary, mentioned how it could have a counter impact:

In case of individuals reporting severe suicidal ideations during research, referring them for professional help is for their betterment and we do that, but often this help-seeking requires them to revisit their emotional pain multiple times in front of different professionals. This adds to their distress and makes them hopeless and just wary of the entire process. (RF4)

Similar issues were shared by the clinicians during FGD-2. Most psychologists and psychiatrists agreed on how adhering to the treatment protocol (such as hospital admission or asking the family to constantly accompany the survivor) was critically valuable for the patients' well-being. At the same time it could heighten the stigmatisation faced by them and curtailed their freedom. Additionally, for some patients, it could lead to a change in the personal, social, and occupational dynamics of their lives, thus strengthening the triggering factors of their attempt: 
Table 1: Results indicating the subthemes, themes and operational definitions of the ethical dilemmas encountered by researchers and clinicians working with suicide survivors

\begin{tabular}{|c|c|c|c|}
\hline Themes & Operational Definition & Subthemes & $\begin{array}{l}\text { Participant } \\
\text { Identity }\end{array}$ \\
\hline \multirow{4}{*}{$\begin{array}{l}\text { Struggling with the } \\
\text { incongruity of harm within } \\
\text { benefit }\end{array}$} & \multirow{4}{*}{$\begin{array}{l}\text { The fear of causing } \\
\text { unintentional harm while } \\
\text { complying with the } \\
\text { standardised processes } \\
\text { aimed at providing benefit to } \\
\text { the individual. }\end{array}$} & $\begin{array}{l}\text { Referring to multiple stakeholders versus distress of } \\
\text { participant }\end{array}$ & Researchers \\
\hline & & $\begin{array}{l}\text { Standard intervention process versus doubt over } \\
\text { intervention effectiveness }\end{array}$ & Researchers \\
\hline & & $\begin{array}{l}\text { Reinforcing symptomatology in patients by adhering to } \\
\text { standardised protocol }\end{array}$ & $\begin{array}{l}\text { Researchers + } \\
\text { Clinicians }\end{array}$ \\
\hline & & $\begin{array}{l}\text { Reporting suicidal behaviour for treatment versus } \\
\text { stigmatisation agony }\end{array}$ & Clinicians \\
\hline \multirow{3}{*}{$\begin{array}{l}\text { Difficulty in delineating } \\
\text { boundaries }\end{array}$} & \multirow{3}{*}{$\begin{array}{l}\text { Concern over blurring of } \\
\text { boundaries with having to } \\
\text { resonate with the intense } \\
\text { emotional experience of the } \\
\text { individuals, at the risk of } \\
\text { becoming overly absorbed in } \\
\text { their lives. }\end{array}$} & Dichotomy between researcher and counsellor roles & Researchers \\
\hline & & Navigating the engagement of and with family members & $\begin{array}{l}\text { Researchers + } \\
\text { Clinicians }\end{array}$ \\
\hline & & Heightened involvement due to higher risk to life & $\begin{array}{l}\text { Researchers + } \\
\text { Clinicians }\end{array}$ \\
\hline \multirow{4}{*}{$\begin{array}{l}\text { Self-doubt in one's } \\
\text { professional competence }\end{array}$} & \multirow{4}{*}{$\begin{array}{l}\text { Overwhelmed by relating to } \\
\text { the experiences and } \\
\text { emotions of the individual } \\
\text { with suicidal thoughts, } \\
\text { leading to self-doubt in one's } \\
\text { abilities }\end{array}$} & Fluctuating engagement with participant due to burnout & Researchers \\
\hline & & $\begin{array}{l}\text { Guilt, anxiety and unsettling of faith due to losing a } \\
\text { person to suicide }\end{array}$ & $\begin{array}{l}\text { Researchers + } \\
\text { Clinicians }\end{array}$ \\
\hline & & $\begin{array}{l}\text { Discrepancy between subjective beliefs and professional } \\
\text { expectations }\end{array}$ & $\begin{array}{l}\text { Researchers + } \\
\text { Clinicians }\end{array}$ \\
\hline & & Uncertainty in assessing suicidal crisis & $\begin{array}{l}\text { Researchers + } \\
\text { Clinicians }\end{array}$ \\
\hline \multirow{3}{*}{$\begin{array}{l}\text { Nature of suicide - Straining } \\
\text { the limits of confidentiality }\end{array}$} & \multirow{3}{*}{$\begin{array}{l}\text { Escalated fear over possible } \\
\text { breaching of confidentiality } \\
\text { of individual with suicidal } \\
\text { thoughts, while dealing with } \\
\text { psycho-social and } \\
\text { environmental limitations }\end{array}$} & $\begin{array}{l}\text { Maintaining patients' confidentiality versus informing } \\
\text { family stakeholders }\end{array}$ & Clinicians \\
\hline & & Client autonomy versus family vigilance & $\begin{array}{l}\text { Researchers + } \\
\text { Clinicians }\end{array}$ \\
\hline & & Compromising confidentiality due to infrastructural limits & $\begin{array}{l}\text { Researchers + } \\
\text { Clinicians }\end{array}$ \\
\hline \multirow{3}{*}{$\begin{array}{l}\text { Working with structural } \\
\text { limitations }\end{array}$} & \multirow{3}{*}{$\begin{array}{l}\text { Difficulty in doing justice to } \\
\text { vulnerable individuals, while } \\
\text { working within } \\
\text { compromised functional } \\
\text { limitations. }\end{array}$} & $\begin{array}{l}\text { Providing sufficient time uniformly to all patients and } \\
\text { participants }\end{array}$ & $\begin{array}{l}\text { Researchers + } \\
\text { Clinicians }\end{array}$ \\
\hline & & Defined protocols yet procedural uncertainties & $\begin{array}{l}\text { Researchers + } \\
\text { Clinicians }\end{array}$ \\
\hline & & Accounting for safety of the patient or oneself & $\begin{array}{l}\text { Researchers + } \\
\text { Clinicians }\end{array}$ \\
\hline \multicolumn{4}{|c|}{ \#Note: Researchers = 2 clinical psychologists and 3 psychiatric social workers [Research Fellows at ICMR] } \\
\hline Clinicians $=4 \mathrm{~S}$ & lents in Psychiatry and 4) & g Clinical Psychologists [mental health profes & \\
\hline
\end{tabular}

Before meeting us, the patient was roaming around freely in the mall or working without any occupational restriction, but now if we write it on paper (that they are under treatment for suicidal behaviour) or admit a patient, the patient faces problems, and if we don't, that is wrong and risky on our part .... It harms their social status and reconciliation with the

$$
\text { society, but it's part of the treatment protocol. (SR3) }
$$

Hence whether the treatment protocol designed to promote the well-being of an individual also inflicts a certain degree of harm upon them, it is a concern for both the researchers and the clinicians. However, due to the short duration and 
more stringent norms of involvement (due to their research protocol) RFs find it more difficult to maintain contact and support with vulnerable research participants. Hence this becomes a predominant concern for the researchers, more prominently than for the clinicians.

\section{Theme 2: Difficulty in delineating boundaries}

As the researchers shared the need for a "sort of therapeutic alliance" because of the delicate nature of exploration with individuals suffering from suicidal thoughts, they also admitted to feeling apprehensive about violating the established research boundaries. All five researchers unanimously agreed that they faced role confusion due to the pressure to either play the role of a counsellor or risk coming across as an emotionally distant researcher. This may be because the research was interventional in nature and their participants' expectations would be for therapeutic interventions even if the nature of the research was specifically explained during the consent process. One of the narratives exemplifying this concern was:

When your participant (suicide attempt survivor) is so distressed that they are actually contemplating death, sometimes you have to step beyond the research protocol, and often, the participant (suicide attempter survivor) wants you to counsel them, become their therapist; but you can't do that, yet you also can't be just a mechanical researcher... drawing that line becomes tough. (RF3)

However, the blurring of boundaries was an ethical dilemma encountered by practising clinicians as well. They tended to inadvertently become overinvolved in or paternalistically protective of their participant/client, as illustrated in these statements:

The duty to protect is very high with such clients, so at times the boundary becomes blurred ... you get over-involved with the client or allow the client to be dependent on you for quite some time because the anxiety about losing the client is very high. (CP1)

In critical cases, you always feel protective ... It is coming from instinct, not as a clinician or a researcher. Just the thought that this person might die, makes you too overinvolved at times, and in that process, it becomes difficult to focus only on the research objective. (RF2)

The researchers and clinicians both described how they found themselves at a cross-roads with psychosocial issues embedded in their participant's/client's familial circumstances. Consequently, they faced the unending expectations of their participants/clients, and those of their participant's/client's family members, to intervene and engage beyond their customary capacities, as described below:

Suicide is a psychosocial problem. Your participants (suicide attempter survivors) want you to repeatedly psycho-educate their family or to mediate with them about these social issues, and the family members also want their plight to be addressed! If it is an individual-based intervention, you cannot scatter the research completely, you cannot be getting involved in those dynamics, but your participants expect that. (RF1)

You tell the family to be vigilant with the participants (suicide attempter survivors), especially in case of those presenting high suicide risk, but that at times also frustrates the participant. At the same time, the participant, her/his family, they look up to you for betterment.... Then, if participant reattempts or acts out in a self-injurious manner due to the frustration of being monitored by her/his family ... then you not only feel guilty yourself, but the family's guilt is also on you because you asked them to be vigilant in the first place. (CP 2)

\section{Theme 3: Self-doubt in one's professional competence}

One of the major dilemmas faced by both the researchers and clinicians was that, despite training and trust in one's clinical and information competence, losing a participant/client/patient to suicide impacted them personally as well as professionally, creating self-doubt about their own competence.

When we lose our participant (suicide attempter) due to suicide during the research process ... as a researcher, you end up feeling shaken, and continuing with the research with same faith is tough. (RF1)

When coming for treatment itself becomes a stressor, and in that stress, they re-attempt ... it makes you question yourself. It makes you feel ashamed and guilty. (CP3)

The aftermath of a patient's/ research participant's reattempt or completed suicide, thus surfaced in the form of emotional upheaval, with varied emotions like shock, anger, sadness, guilt, anxiety, self-blame, and doubts over one's competence. Additionally, for the researchers/clinicians, the incongruity between one's subjective beliefs and professional expectations also evoked reservations over one's competence. In cases of suicide, as professionals, they confessed that certain issues were more important for them and they could not withhold the spilling over of their personal beliefs. This was highlighted by one of the researchers and clinicians and the others concurred:

For instance, I had an orphaned client; she was being physically and sexually abused by her uncle, who was the sole breadwinner for her family. She was not educated enough to support herself. She was feeling totally hopeless, and in despair, she attempted. I felt outraged and wanted her to report the atrocity; yet I felt as helpless as her. In situations like these, when as a professional, it is our job to widen their perspective and somewhere we too end up feeling that there is no alternative available. Then you feel extremely guilty, ashamed. (CP1) 
Another situation strengthening the underpinning ethical dilemma of competence was the fear of inaccurately assessing the severity of suicide crisis among their patients/ research participants as was shared by one of the clinicians:

There are times when a patient repeatedly mentions (suicidal) ideations but has no evident self-harming behaviour, and even the family members start taking it casually, so the patient feels that no one is caring and, in that disappointment, they attempt (again). Such cases can be very tricky; these unexpected cases can sometimes shock you and pull you down. (SR4)

Lastly, the researchers acknowledged feelings of incompetence when the frequent encounters with their participants whose lives were at risk with suicidal thoughts became overwhelming and induced feelings of exhaustion and emotional fatigue. As illuminated in the narratives of all the researchers,

The researcher's mental health status or preparedness also matter. I have already worked extensively with two-three participants (suicide attempt survivors) ... I am mentally exhausted, working with the next participant on the same day then seems challenging. This again makes you feel guilty for not being good enough or for not doing enough justice to each person. (RF5)

Hence, when dealing with persons with suicidal thoughts/ behaviour, the likelihood of blurring of professional and personal boundaries, risks of emotional engagement as well as ambiguity, are greater.

\section{Theme 4: Nature of suicide - Straining the limits of confidentiality}

At times, it becomes difficult to maintain confidentiality for legal reasons, as well as to harness support and involvement of the family members, especially in life-threatening cases, as disclosed by the clinicians during the discussion:

If the patient comes alone ... it is important to inform their family as well, but at the same time, it means breaking the patient's confidentiality, and you also risk abandonment of the patient by the family. But if you do not disclose, you are putting the patient at risk without building a support system. (SR2)

Further discussions revealed that such a situation is difficult to resolve and presents its own share of complexities and conflicts, especially in the case of adolescents. Both researchers and clinicians shared the dilemma about whether breaking confidentiality would reduce or further escalate the risk of violence and self-harm among the younger participants/patients. For instance, the clinicians explained how disclosing confidential information could threaten the autonomy of their patient and translate into reduced access to help in case of a crisis:

When you find something is injurious or harmful to your patient's well-being, then disclosure to family is important but risky. Even when you try to take your patient into confidence, they agree to share something vital only if you don't tell anyone else. Now you are stuck!! If you insist on disclosing to the family, you are preventing your patient from giving significant information, and if you agree to your patient's precondition, you are withholding dangerous information from close ones! (CP2)

In addition, external factors, like infrastructure limitations also led to the breach of confidentiality. For instance, one of the researchers highlighted:

There are times, due to the spatial restraints, we are unable to ensure adequate privacy for each of our participants (suicide attempt survivors), and these participants feared being overheard or felt constrained due to the number of people around ... but we do not have much of a choice in those circumstances. (RF4)

Thus, despite awareness of the guiding principles of confidentiality, the researchers/clinicians felt the need to be constantly mindful of the associated risks.

\section{Theme 5: Working with structural limitations}

One of the major limitations as shared by the researchers and clinicians alike, was lack of adequate time and space. In the case of individuals presenting with risk of suicide, the constraints in providing more time and heightened sensitivity were further challenged:

Because of social, legal concerns, these patients are not always open about their symptoms ... they are sceptical. So, you must make them extremely comfortable, and that takes a lot of time, which most of the practitioners do not have. This can be a hindrance in the quality of care. (CP2)

Another difficulty is having to follow the standardised protocol, which can hinder the process of acquiring clarity about the patient's/client's positionality and provide adequate help to him/her. For instance, the researchers and clinicians revealed:

Research is outcome-oriented and has its fixed modalities. The structured intervention, strategies, and modules help, but at the same time, there are cases where our participant's (suicide attempter's) environment doesn't adequately support the intervention strategy or when, as a researcher, you feel help could be offered differently, but that means going beyond the protocol, and you can't ... then you feel stuck at times. (RF2)

We have our protocols, but in case of some patients, when there is a lack of clarity over the diagnosis, we admit the patient. But if the patient runs away and attempts, or in some cases, where the patient comes alone, the patient threatens to attempt and then absconds, who will be held responsible? How to intervene and how much to? (SR2)

The protocols also spawned a dilemma particularly in terms of confidentiality. During the discussion, it was established 
that in case maintaining confidentiality operated as a risk to their occupational status, it became critical for these professionals (researchers/clinicians) to breach it. The following narratives shed light on the dilemma they encountered:

In cases of suicide, there can be a lot of legal issues as well ... so, if there is a serious danger, we have to break confidentiality because we have to protect ourselves legally, or we can be sued any time. (RF3)

When a patient comes with an attempt, we psycho-educate the family and always write "high-risk precautions" as a blanket term, expecting high vigilance from the family. Now, the patient can feel bothered and frustrated to have someone on their back all the time, and that can induce further stress. I realize this, but ... to some extent, for my own safety, I will still write it. (CP4)

\section{Discussion}

To our knowledge, this is one of a few papers examining the ethical dilemmas of those who intervene in suicidal behaviour - whether as clinicians or as researchers. The ethical questions began as discussions among authors and culminated in two FGDs; FGD1 comprising researchers and FGD 2 comprising clinicians. Members of FGD 2 dealt with suicide survivors on a regular basis, while FGD1 members adhered to strict inclusion criteria $(20,21)$. The two separate FGDs with researchers and clinicians revealed that, for young mental health professionals, suicide research and treatment can involve numerous ethical challenges and dilemmas.

Based on the nature of research into suicide and the management strategies adopted, ethical implications and challenges encountered by such professionals can vary. We tried to address some universal and critical ethical dilemmas and concerns faced by young professionals. One of the most prominent and persistent challenges was the uncertainty about unintended yet plausible harm to their participants/ patients while working towards providing benefits. In concurrence with previous studies, we found that fear and apprehension about reinforcing symptomatology during risk assessment prevailed amongst the researchers (12). This fear was deeper among researchers engaged in online or telephonic intervention with individuals presenting an imminent risk of suicide, as well as with researchers in our study (25). Further, in order to prevent a role overlap researchers voiced their inability to satisfactorily respond to the therapeutic needs of their research participants. This compromised the ideal management of these vulnerable individuals $(26,27)$.

The dilemma of beneficence and non-maleficence persisted for the clinicians namely psychiatrists and clinical psychologists as well. For the clinicians, adhering to the treatment protocol of mandatory hospitalisation for acute and recurrent suicides or mentioning suicidality on medical reports risked triggering suicidal behaviour in some patients.
Since stigma and discrimination in the form of social exclusion and reduced opportunities for those labelled mentally ill has been found to be high in India (28), the clinicians feared they might further harm the social support system and self-esteem of an at-risk individual. Some reports mention that standardised measures sometimes result in stigma and labelling of suicide survivors leading to irregularity in availing of mental healthcare services(29).

Difficulty in demarcating boundaries between their professional and personal roles was another ethical dilemma encountered by both groups. Defining boundaries is an integral aspect of any research or therapeutic relationship. These boundaries aid in equalising the power dynamics and preventing any possible exploitation of the research participants/patients. The struggle to refrain from acceding to the therapeutic expectations of their participants, while still facilitating a safe environment, conducive to sharing one's stories, was a dilemma particularly experienced by the researchers here and has also been reported in other studies $(26,30)$.

Relational boundaries for both researchers and clinicians were blurred because of the primary need to protect the individual and prevent suicide. The urge to ensure the safety of their participant/patient and to eliminate the chances of suicidal behaviour increases the sense of accountability for a researcher as well as for a clinician $(13,31)$. However, adhering to these boundaries was more difficult when the burden of responsibility to care for their research participant/patient weighed primarily upon them. As a result, they felt pushed to get involved with a participant's family members and their consequent expectations $(32,33)$. More so because, in countries like India, mental healthcare professionals are looked up to and expected to be directive, thereby increasing the responsibility thrust upon them $(34,35)$.

Blurred boundaries not only posed an ethical dilemma in themselves, but spawned an additional dilemma of diminished confidence in one's own competence. Possessing and demonstrating professional competence is a vital skill for any mental health professional. However, in the case of individuals at risk of suicide, what constituted competency and how effectively the professionals could utilise their skills became a challenging issue. The perceived sense of selfimposed accountability for saving their research participant's/ patient's life, when not satisfactorily met, gave rise to selfloathing and insecurities. The frequent contact, deep immersion in their narratives and heightened feelings of personal responsibility, resulted in feelings of shame, guilt, anxiety, and hypervigilance. This occurred especially in the case of the loss of an individual to suicide, blurring the personal and professional boundaries $(36,37)$. Inability to balance one's personal outlook and beliefs with professional obligations also raised doubts over one's own competence. Even ethics committee members felt that the role of researchers in suicide research is likely to become blurred with those of care givers (12). 
In therapy and research, professionals are often faced with the challenge of having to overlook their own strong emotions. However, in the case of sensitive and vulnerable populations, these competing concerns collide with universal ethical principles $(38,39)$. Our clinicians highlighted the difficulty in maintaining faith and belief (in their own competence) in complex suicide crisis situations. Despite responsible engagement, professional acumen, and rigorous adherence to the principles of risk assessment, the complexity of suicide made it difficult to guarantee suicide prevention for all individuals in all situations $(13,40)$.

This not only generated a fear of legal repercussions for these professionals, but also took a toll on their confidence in their own skill and competence. The reservations over one's capabilities, however, emerged not only from overinvolvement with their participants, but on the contrary, also from their inability to be fully involved with their participant's narratives. Our researchers emphasised how the emotional and mental exhaustion experienced during research impacted their quality of engagement with their participants, thereby generating doubts over their own competency. Other authors have also emphasised how recurrent exposure to such a vulnerable population accumulates, and emotionally exhausts the researcher (41, 42).

The intricacies and complications linked with suicide further strain the basic principles of ethics such as those of confidentiality. This was predominantly experienced by the clinicians who struggled to maintain the patient's confidentiality while simultaneously garnering familial support and initiating specialised treatment. This sometimes obliged them to breach the confidentiality of their patient and escalated their fear of exposing their patient to risks of abandonment, ostracisation, and maltreatment by family members $(28,43)$. Also, the inviolability of life as an absolute truth positions the researcher to prevent suicide at all costs (44). Hence, maintaining confidentiality and honouring the autonomy of their client presented a looming risk of possible self-harm in some clients, especially adolescents and young adults. In such cases breaking confidentiality to ensure the client's safety by disclosure to a family member was deemed essential. Extending this dilemma, a breach in confidentiality posed the risk of either their client withholding information and/or the possibility of the family restricting their freedom $(13,17,45)$. However, in India, maintaining confidentiality also becomes challenging due to resource constraints like infrastructural inadequacies and limited manpower relative to the number of ailing individuals $(46,47)$.

Both clinicians and researchers felt maintaining confidentiality was difficult because it came at the cost of disregarding the structural limitations of one's work. For instance, certain circumstances, like failure to foresee a possibility of self-harm or suicidal behaviour, made them fearful of possible lawsuits and other legal issues. Thus, it was necessary for them to safeguard themselves and report the presence of any suicidal behaviour at the earliest (29). Similarly, there were other structural limitations as well as procedural accountability such as insufficiency of time or standardised protocols $(48,49)$ which in some circumstance generate a feeling of powerlessness. Consequently, clinicians as well as researchers found themselves facing complex ethical dilemmas while attempting to deliver care to their patients/clients and participants.

In conclusion, research into suicide is required, and immediate clinical intervention after a suicide attempt is necessary. Yet it may come at the cost of self-doubt for the professionals concerned. In such circumstances, establishing boundaries while creating a support system for researchers and clinicians should be as essential as creating protocols to help the individual with suicidal thoughts.

\section{Limitations}

This research was undertaken at the psychiatry department of a single institute. Although it is a government-managed, teaching tertiary care centre with a high patient load, yet bias may have crept in, both in the type of patients who avail such services and in the professionals who train here. Nevertheless, considering the published literature, their observations echoed those described by other authors. Secondly, the sample size of the study was small, and though it helps shed light on critical issues in practice, carrying out further studies with a larger sample size is desirable.

\section{Conclusion and implications}

Despite acknowledging the sensitive, intrusive and complex nature of interactions intrinsic to suicide research and treatment, ethical issues and emotional burden encountered by researchers and clinicians during such work have been largely neglected, especially in India The current study sheds light on some of these concerns such as an enhanced sense of responsibility intensified by fear, difficulty in establishing boundaries, maintaining confidentiality, and ensuring benefit while avoiding harm. Although we highlighted the ethical, legal, and practical constraints which bedevil suicide research and treatment, it is essential to explore the resolution mechanisms adopted by the professionals for navigating these challenges. Furthermore, as emphasised by the World Health Organisation(1) it is equally important to recognise and build mechanisms which encourage communities, families, social workers, etc, to work together in bottom-up processes to enable mental health professionals to better implement protective and care management strategies for those presenting a risk of suicide (1).

In conclusion, discussions about striking a balance between the duty to protect an individual and adhering to professional limitations, reflections about one's perceptions of suicide impacting the nature of interaction with one's participants/clients, as well as adapting to the dynamic changes and intricacies inherent in suicide cases can lead to the development of better and more flexible guidelines for 
interventions. These insights can aid in cultivating a reflective platform for healthcare professionals and help develop better ethics training modules pertaining to the complex paradigm of suicide. Lastly, the findings of this study also suggest that capacity building programmes which are sensitive to our psychosocial structures and mental health support services need to be adapted and implemented for those professionals engaged in working with similar mental health problems.

\section{Acknowledgements}

The salary of Triptish Bhatia was supported by the grant 'CrossFertilized Research Training for New Investigators in India and Egypt' (D43 TW009114, HMSC File No. Indo-Foreign/35/M/2012NCD-1, funded by the Fogarty International Centre, National Institutes of Health (NIH)); and National Coordination Unit funded by ICMR (file no. 5/4/-4/151/M/2017/NCD-1 to SND). We are also thankful to the ongoing research project 'A multi-centric randomized controlled trial to evaluate the efficacy of telephone-based psychosocial interventions on future suicide risk in suicide attempters (Trial REF/2018/07/020799, CTRI/ 2018/09/015758 to RPB). NIH and ICMR had no role in the design and conduct of the study; collection, management, analysis, and interpretation of the data; preparation, review, or approval of the manuscript; and decision to submit the manuscript for publication.

Special thanks to all the FGD participants. Their valuable involvement, active participation, and meaningful insights were critical for the constructive completion of this study.

\section{Conflict of interest: None declared for any of the authors.}

\section{References}

1. World Health Organization. Suicide. Geneva:WHO; 2019. [cited 2020 Dec 4]. Available from: https://www.who.int/news-room/fact-sheets/ detail/suicide.

2. Thippaiah SM, Nanjappa MS, Math SB. Suicide in India: A preventable epidemic. Indian J Med Res. 2019;150(4):324-7.

3. Hendin H, Phillips MR, Vijayakumar L, Pirkis J, Wang H, Yip P, et al., editors. . Suicide and Suicide Prevention in Asia [Internet]. Geneva: World Health Organization:. 2008 [cited 2020 Dec 4]. Available from: https://www.who.int/mental_health/resources/ suicide prevention asia.pdf

4. Hom MA, Podlogar MC, Stanley IH, Joiner TE. Ethical issues and practical challenges in suicide research. Crisis. 2016 Aug 26;38(2):10714.

5. Institute of Medicine (US) Committee on Pathophysiology and Prevention of Adolescent and Adult Suicide. Goldsmith SK, Pellmar TC, Kleinman AM, Bunney WE , editors.. Reducing Suicide: A National Imperative. Washington (DC): National Academies Press (US); 2002.

6. Vijayakumar L. Indian research on suicide. Indian J Psychiatry. 2010;52(Suppl 1):S291-6.

7. Thummapoi O, Park T, Jackson M, Barton S. Methodological Challenges faced in doing research with vulnerable women: reflections from fieldwork experiences. Int J Qual Methods. 2019;18:1-11.

8. Micanovic LS, Stelko S, Sakic S. Who else need protection? Reflecting on researcher vulnerability in sensitive research. Societies. 2019;10(1).

9. Abley C, Bond J, Robinson L. Improving interprofessional practice for vulnerable older people: Gaining a better understanding of vulnerability. Care. 2011;25(5):359-65

10. Levine C, Faden R, Grady C, Hammerschmidt D, Eckenwiler L, Sugarman J, et al. The limitations of "vulnerability" as a protection for human research participants. Am J Bioethics. 2004;4(3):44-9.

11. Roberts LW, Berk MS, Lane-McKinley K. Ethical Considerations in Research on Suicide Prediction: Necessity as the Mother of Invention. JAMA Psychiatry. 2019;76(9):883-4.
12. Lakeman $R$, Fitzgerald $M$. The ethics of suicide research. Crisis 2009;30(1):13-9.

13. Nugent AC, Ballard ED, Park LT, Zarate CA. Research on the pathophysiology, treatment, and prevention of suicide: practical and ethical issues. BMC Psychiatry. 2019;19(1):332.

14. Andriessen K, Reifels L, Krysinska K, Robinson J, Dempster G, Pirkis J. Dealing with Ethical Concerns in Suicide Research: A Survey of Australian Researchers. Int J Environ Res Public Health. 2019;16(7).

15. Hendin H, Haas AP, Maltsberger JT, Koestner B, Szanto K. Problems in psychotherapy with suicidal patients. Am J Psychiatry. 2006;163(1): 67-72.

16. Politis AN, A. K. Registered Australian psychologists' responses to ethical dilemmas regarding medicare funding of their services. Aust Psychol. 2013;48(4):281-9.

17. Bhola P, Sinha A, Sonkar S, Raguram A. Ethical dilemmas experienced by clinical psychology trainee therapists. Indian J Med Ethics. 2015;12(4):206-12.

18. Hagen J, Hjelmeland $\mathrm{H}$, Knizek BL. Connecting with suicidal patients in psychiatric wards: Therapist challenges. Death Studies. 2017;41(6): 360-7.

19. Jain S, Kuppili PP, Pattanayak RD, Sagar R. Ethics in Psychiatric Research: Issues and Recommendations. Indian J Psychol Med. 2017;39(5):558-65.

20. Neuman WL.. Social research methods: Quantitative and qualitative approaches (6th ed.). Pearson Education; Dorling Kindersley (India); 2016.

21. Beniwal RP, Sreedaran P, Chari U, Ashok MV, Bhatia T. Protocol of a multi-centric randomized controlled $t$ rial to evaluate eficacy of telephone-based psychosocial interventions on future suicide risk in suicide attempters. Indian J Psychol Med. 2020;42(6):39-45.

22. Sreedaran P, Beniwal RP, Chari U, Smitha TS, Vidhya Shree SV, Gupta V, et al. A Randomized Controlled Trial to Assess Feasibility and Acceptability of Telephone-Based Psychosocial Interventions in Individuals Who Attempted Suicide. Indian J Psychol Med. 2020;42.

23. Hedlund de-Witt NH. Coding: An overview and guide to qualitative data analysis for integral researchers. Integral Research Center Resource Paper No. 1; 2013[cited 2021 Dec 8] Available from: https:// www.academia.edu/9864164/

Coding_An_Overview_and_Guide_to_Qualitative_Data_Analysis_fo r_Integral_Researchers

24. Braun V, Clarke V. Using thematic analysis in psychology. Qual Res Psychol. 2006;3.:77-101

25. Bailey E, Mühlmann C, Rice S, Nedeljkovic M, Alvarez-Jimenez $M$, Sander $L$, et al. Ethical issues and practical barriers in internet-based suicide prevention research: a review and investigator survey. $B M C$ Med Ethics. 2020;21(1):37.

26. Thompson A, Russo K. Ethical Dilemmas for Clinical Psychologists in Conducting Qualitative Research. Qual Res Psychol. 2012;9:32-46.

27. McKenzie SK, C. L, Jenkin G, Collings S. Ethical considerations in sensitive suicide research reliant on non-clinical researchers. Research Ethics. 2016.

28. Böge K, Zieger A, Mungee A, Tandon A, Fuchs LM, Schomerus G, et al. Perceived stigmatization and discrimination of people with mental illness: A survey-based study of the general population in five metropolitan cities in India. Indian J Psychiatry. 2018;60(1):24-31.

29. Ho AO. Suicide: rationality and responsibility for life. Can J Psychiatry. 2014;59(3):141-7.

30. Kendall S, Halliday LE. Undertaking ethical qualitative research in public health: are current ethical processes sufficient? Aust N Z J Public Health. 2014;38(4):306-10.

31. Awenat YF, Peters S, Gooding PA, Pratt D, Huggett C, Harris K, et al. Qualitative analysis of ward staff experiences during research of a novel suicide-prevention psychological therapy for psychiatric inpatients: Understanding the barriers and facilitators. PLoS One. 2019;14(9):e0222482.

32. Moerman M. Working with suicidal clients: The person-centred counsellor's experience and understanding of risk assessment. Couns Psychother Res. 2012;12(3).

33. Singh GP. Understanding emotional turmoil and resolution of disturbed family relationship issues in a suicidal patient. Indian J Soc Psychiatry. 2015;31 84-7.

34. Amiya RM, Poudel KC, Poudel-Tandukar K, Pandey BD, Jimba M. Perceived family support, depression, and suicidal ideation among people living with HIV/AIDS: a cross-sectional study in the Kathmandu Valley, Nepal. PLoS One. 2014;9(3):e90959.

35. Venkatesan S. Some Cultural Aspects of Clinical Counselling in Indian Context. Global Journal of Interdisciplinary Social Science. 2016;5 216. .

36. Rossouw G, Smythe E, Greener P. Therapists' Experience of Working 
with Suicidal Clients. Indo-Pacific Journal of Phenomenology. 2011;11(1):1-12.

37. Howe E. Five ethical and clinical challenges psychiatrists may face when treating patients with borderline personality disorder who are or may become suicidal. Innov Clin Neurosci. 2013;10(1):14-9.

38. Haeny AM. Ethical Considerations for Psychologists Taking a Public Stance on Controversial Issues: The Balance Between Personal and Professional Life. Ethics Behav. 2014;24(4):265-78.

39. Handlesman, M.M., Knapp SJ, Gottlieb MC. Ethical dilemmas in Psychotherapy: Positive approaches to decision making. American Psychological Association 2015.

40. Jacobs DG, Baldessarini RJ, Conwell Y, Fawcett JA, Horton L, Meltzer $\mathrm{H}$, et al. Practice guideline for the Assessment and Treatment of Patients With Suicidal Behaviors. In: on Apasc, guidelines p, editors. 2010.

41. Boden ZV, Gibson S, Owen GJ, Benson O. Feelings and Intersubjectivity in Qualitative Suicide Research. Qual Health Res. 2016;26(8):1078-90.

42. Saigle V, Racine E. Ethical challenges faced by healthcare professionals who care for suicidal patients: a scoping review. Monash Bioeth Rev. 2018;35(1-4):50-79.
43. Gibson S, Benson O, Brand SL. Talking about suicide: confidentiality and anonymity in qualitative research. Nurs Ethics. 2013;20(1):18-29.

44. Liégeois A, Eneman M. Ethical Aspects of the Prevention of Suicide in Psychiatry. Ethical Human Psychology and Psychiatry. 2012;14(2).

45. Noroozi M, Zahedi L, Bathaei FS, Salari P. Challenges of Confidentiality in Clinical Settings: Compilation of an Ethical Guideline. Iran J Public Health. 2018;47(6):875-83.

46. Beltran-Aroca CM, Girela-Lopez E, Collazo-Chao E. Confidentiality breaches in clinical practice: what happens in hospitals?. BMC Med Ethics. 2016;17

47. Bhasin S, Sharma A, Bhatia T, Deshpande SM. How to, or not to intervene- Some Ethical Dilemmas encountered by Mental Health Professionals. Global Bioethics Enquiry. 2019;7(2).

48. Bruffaerts R, Demyttenaere K, Hwang I, Chiu WT, Sampson N, Kessler $\mathrm{RC}$, et al. Treatment of suicidal people around the world. $\mathrm{Br} J$ Psychiatry. 2011;199(1):64-70.

49. Hom MA, Stanley IH, Joiner TE. Evaluating factors and interventions that influence help-seeking and mental health service utilization among suicidal individuals: A review of the literature. Clin Psychol Rev. 2015;40:28-39. 